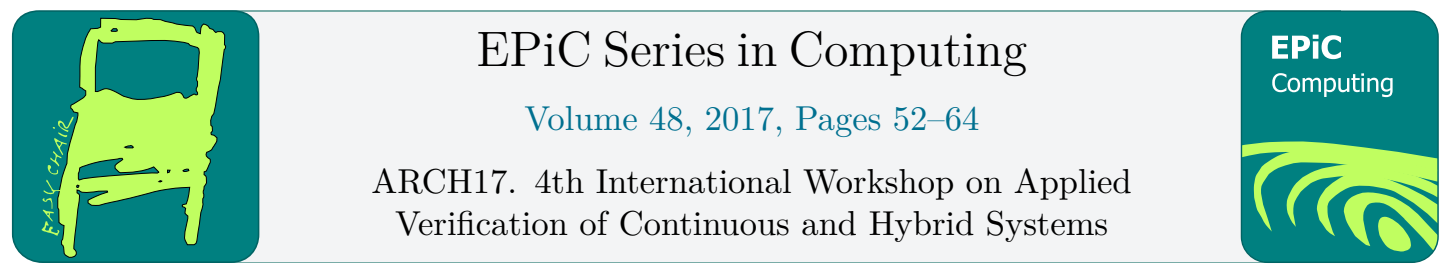

\title{
Reachability Analysis of Transformer-Isolated DC-DC Converters (Benchmark Proposal)
}

\author{
Omar A. Beg ${ }^{1}$, Ali Davoudi ${ }^{1}$, and Taylor T. Johnson ${ }^{2}$ \\ 1 University of Texas at Arlington, Arlington, Texas, USA \\ omar.beg@mavs.uta.edu, davoudi@uta.edu \\ 2 Vanderbilt University, Nashville, Tennessee, USA \\ taylor.johnson@vanderbilt.edu
}

\begin{abstract}
Various mission-critical applications necessarily require a transformer in switching converters to obtain DC isolation between the converters' input and output. Since DC-DC converters are the switching devices, these are modeled as hybrid automata. We present hybrid automaton modeling of two main types of transformer isolated DC-DC converters, namely, flyback and forward converters. We have also catered the non-determinism for both. We use HyST (Hybrid Source Transformation) tool to automatically generate the models in SpaceEx format, perform reachability analysis, and then automatically convert the models into Mathworks Simulink Stateflow (SLSF) using HyST. Thus we demonstrate effectiveness of HyST tool in the model-based design process. The HyST user needs not to manually construct or modify the models thus saving significant amount of time and efforts.
\end{abstract}

Category: academic Difficulty: medium

\section{Context and Origins}

DC-DC converters are the power electronics devices that are extensively used in automotives, industrial, and defense related applications and their mission-critical nature necessitates formal verification prior implementation. Over the period, there has been a drastic rise in power electronics-related safety recalls in the automotive industry. For example, the main cause for recall of around 700,000 Toyota Prius cars in 2014 was attributed to an error in the interaction between a boost converter and its software controller [11]. Likewise, more than 100,000 Toyota Prius cars were recalled due to an inverter failure [12]. Therefore, this mission-critical domain would require significant confidence in the modeling accuracy. This can be ensured through reachability analysis $[1,6,7]$. We present two potential benchmarks related to transformerisolated DC-DC converters for hybrid verification research community. Transformer isolation is implemented by introducing a transformer at the converter input. In addition to the electrical isolation between the input and the output, transformer-isolated DC-DC converters have some other advantages compared to their non-isolated counterparts such as high efficiency and low 
manufacturing cost [4]. Due to their advantages, these are preferred for the DC-DC applications in industrial and defense-related control/communication systems and distributed power networks. This work is based on hybrid automaton modeling of two main types of transformerisolated DC-DC converters, i.e., flyback converter and forward converter. This is a series of benchmarks [6-8] that are being developed to benefit from formal verification prior to field implementation and deployment.

Flyback converter may be regarded as a transformer-isolated buck-boost converter, whereas, forward converter acts as a transformer-isolated buck converter. We develop hybrid automaton models of flyback and forward converters, and use SpaceEx [5], a reachability analysis tool, to compute the over-approximated sets of reachable states ${ }^{1}$. This is a classical fixed point computation tool that operates on symbolic states.

We also use HyST (Hybrid Source Transformation) tool [2] to automatically convert the hybrid automaton models developed in SpaceEx to MathWorks Simulink/Stateflow (SLSF) models ${ }^{2}$. It is a source-to-source translation tool that takes input in the SpaceEx model format, and translates it to the formats of HyCreate, Flow*, dReach, C2E2, Passel 2.0, and HyComp. In addition, it is also used to automatically generate the hybrid automaton models in SpaceEx format as per user-defined parameters and settings. Additional tool support is being added from time to time. Verification and validation research community may use HyST to automatically transform the hybrid automaton models in SpaceEx format to other formats and perform reachability analysis using aforesaid model checking tools.

\section{Hybrid Automaton Modeling of Transformer-Isolated DC-DC Converters}

We present the hybrid automaton modeling of flyback and forward converters in this section. We assume that transformer losses are negligible with perfect coupling among the windings. The transformer is modeled using a parallel magnetizing inductance $L_{m}$ at the input side, called the primary side. The winding towards the output is called the secondary winding. Let $n$ be the turns ratio of primary to secondary windings. Let $v_{1}$ and $v_{2}$ be the voltage across primary and secondary windings, $i_{1}$ and $i_{2}$ be the respective currents, and let $n_{1}$ and $n_{2}$ be the respective number of turns. Following relations hold for an ideal transformer

$$
\frac{v_{1}}{n_{1}}=\frac{v_{2}}{n_{2}},
$$

and

$$
n_{1} i_{1}=n_{2} i_{2}
$$




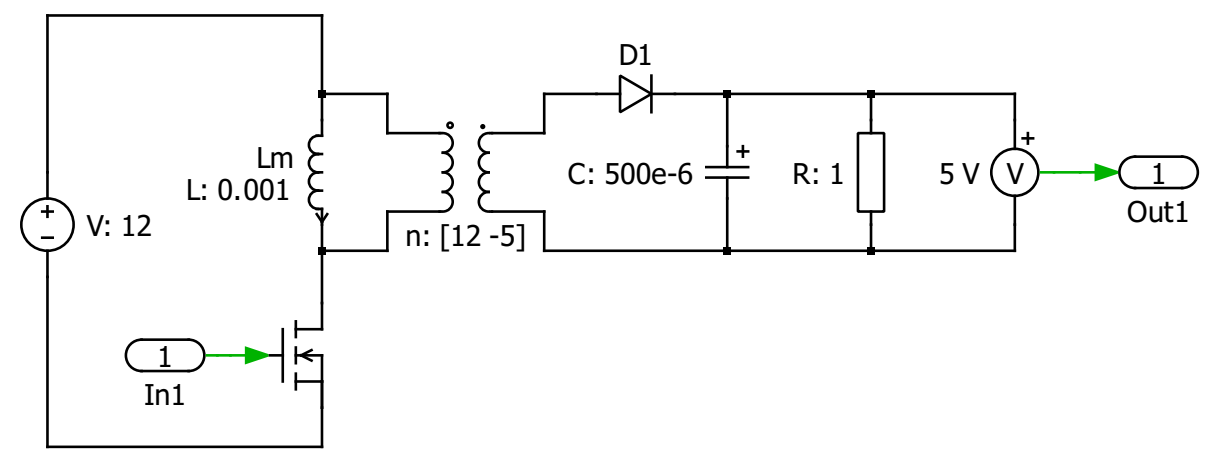

Figure 2.1: Schematic diagram of the flyback converter.

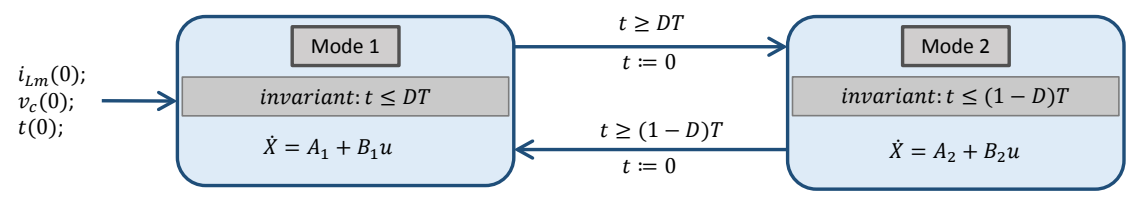

Figure 2.2: Hybrid automaton model for flyback converter.

\subsection{Flyback Converter Modeling}

We consider the flyback converter in open-loop configuration as shown in Figure 2.1 exported from PLECS software [9], a power electronics circuit simulator. The switching is realized by the MOSFET switch and the diode $D_{1}$. The state variables are defined by the voltage across the capacitor $v_{C}$, and current through the magnetizing inductor inductor $i_{L m}$. The MOSFET switch is operated by a pulse generator of constant duty cycle $D$, over the switching time period $T$. The operation of this circuit is dependent upon the state of the MOSFET switch, i.e., being $\mathrm{ON}$ and $\mathrm{OFF}$, resulting into two modes:

1. Mode 1: In this mode, the MOSFET switch is ON during the switching cycle $0<t \leq D T$, wherein, the input DC voltage $V_{i n}$ is connected to the primary of the transformer. This induces the current in the secondary winding in opposite polarity to reverse bias the diode (setting it to OFF state). In this mode, the primary of the transformer is charged, wheres, the diode acts as an open switch causing the capacitor to discharge through the load resistance. We model the MOSFET switching loss by a series resistor $r_{s w}$. The ordinary differential equations (ODEs) for $i_{L m}$ and $v_{C}$ for this mode are formed using conventional Kirchoff' voltage law (KVL) and Kirchoff's current law (KCL). Applying KVL on the left loop gives

$$
\frac{d i_{L m}}{d t}=\frac{r_{s w}}{L_{m}} i_{L m}+\frac{V_{i n}}{L_{m}}
$$

whereas, applying KVL on the loop containing $R$ and $C$ gives

$$
\frac{d v_{C}}{d t}=\frac{1}{R C} v_{C} .
$$

\footnotetext{
${ }^{1}$ The tool is available online from the SpaceEx website at: http://spaceex.imag.fr/.

${ }^{2}$ The executable models are included on the ARCH website and are also available online from the HyST website at: http://verivital.com/hyst/.
} 
The state space matrices, during the switching cycle $0<t \leq D T$, are thus given by

$$
A_{1}=\left[\begin{array}{cc}
\frac{r_{s w}}{L_{m}} & 0 \\
0 & \frac{1}{R C}
\end{array}\right], B_{1}=\left[\begin{array}{c}
\frac{1}{L_{m}} \\
0
\end{array}\right], X=\left[\begin{array}{c}
i_{L m} \\
v_{C}
\end{array}\right], u=V_{i n}
$$

2. Mode 2: In this mode, the MOSFET switch is OFF during the switching cycle $D T<$ $t \leq T$, thus the input DC power supply is disconnected from the primary of the transformer. The current in the secondary flows in upward direction hence diode is forward biased (in ON state). We first consider the primary winding loop and apply KVL. Using Equation 2.1, the voltage across the primary is given by

$$
v_{1}=-n v_{C},
$$

such that the negative sign is due to its opposite direction. Applying KVL in the primary winding loop, we obtain following relation for the magnetizing inductor current

$$
\frac{d i_{L m}}{d t}=-\frac{n}{L_{m}} v_{C}
$$

The current through primary winding is the same as current through $L_{m}$. From Equation 2.2 , the current through the secondary winding is given by

$$
i_{2}=n i_{L m}
$$

Consider the node joining $R$ and $C$. The current entering this node is $i_{2}$. Applying KCL on this node, we get

$$
\frac{d v_{C}}{d t}=\frac{n}{C} i_{L m}-\frac{1}{R C} v_{C}
$$

The corresponding state space matrices, during the switching cycle $D T<t \leq T$, are thus given by

$$
A_{2}=\left[\begin{array}{cc}
0 & -\frac{n}{L_{m}} \\
\frac{n}{C} & -\frac{1}{R C}
\end{array}\right], B_{2}=\left[\begin{array}{l}
0 \\
0
\end{array}\right]
$$

We have formulated a hybrid automaton model of flyback converter using the above ODEs as shown in Figure 2.2. The component values used in the model are mentioned in Figure 2.1, and adopted from [9].

\subsection{Forward Converter Modeling}

The forward converter may be regarded as a transformer-isolated buck converter, as illustrated in Figure 2.3 sketched using PLECS [9]. It has a MOSFET switch, and three diodes $D_{1}, D_{2}$, and $D_{3}$ to realize the switching operation. We consider three state variables, i.e, magnetizing current $i_{L m}$, inductor current $i_{L}$, and capacitor voltage $v_{C}$. Let $n_{1}, n_{2}$, and $n_{3}$ be the number of turns in three windings of the transformer. The switching modes depend on the state of the MOSFET switch as well as the fact that whether inductor current $i_{L} \leq 0$ and the magnetizing current $i_{L m} \leq 0$. This results in six different modes as under. 


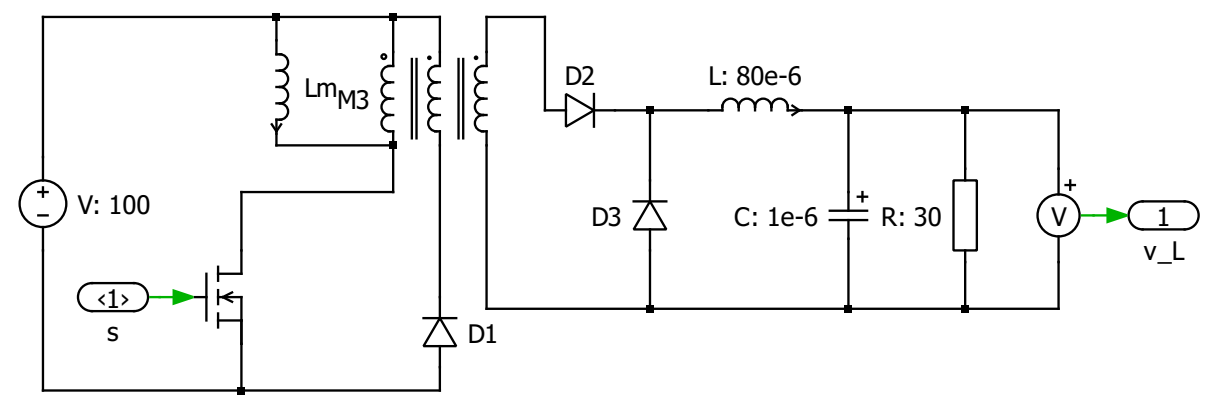

Figure 2.3: Schematic diagram of forward converter.

1. Mode 1: MOSFET switch is ON during the switching cycle $0<t \leq D T$, wherein, the input DC voltage $V_{i n}$ is connected to the primary winding of the transformer. This causes $D_{2}$ to become ON, and $D_{1}$ and $D_{3}$ to OFF. Applying KVL to left most loop results in

$$
\frac{d i_{L m}}{d t}=\frac{V_{i n}}{L_{m}}
$$

The voltage across $D_{3}$ is $\frac{n_{3}}{n_{1}} V_{i n}$. Applying KVL to the loop containing $L$ and $C$, results

$$
\frac{d i_{L}}{d t}=\frac{n_{3}}{n_{1} L} V_{i n}-\frac{1}{L} v_{C} .
$$

Consider the node common to $L, C$, and $R$. Applying KCL here results

$$
\frac{d v_{C}}{d t}=\frac{1}{C} i_{L}-\frac{1}{R C} v_{C} .
$$

The corresponding state space matrices are thus given by

$$
A_{1}=\left[\begin{array}{ccc}
0 & 0 & 0 \\
0 & 0 & -\frac{1}{L} \\
0 & \frac{1}{C} & -\frac{1}{R C}
\end{array}\right], B_{1}=\left[\begin{array}{c}
\frac{1}{L_{m}} \\
\frac{n_{3}}{n_{1} L} \\
0
\end{array}\right], X=\left[\begin{array}{c}
i_{L m} \\
i_{L} \\
v_{C}
\end{array}\right], u=V_{i n} .
$$

2. Mode 2: MOSFET switch is OFF during switching cycle $D T<t \leq(1-D) T$ and $V_{\text {in }}$ is disconnected from winding 1 , with $i_{L m}>0$ and $i_{L}>0$. Diodes $D_{1}$ and $D_{3}$ are ON, and $D_{2}$ is OFF. $V_{i n}$ supplies winding 2 and voltage across $L_{m}$ is $-V_{i n} \frac{n_{1}}{n_{2}}$. This results

$$
\frac{d i_{L m}}{d t}=-\frac{n_{1} V_{i n}}{n_{2} L_{m}} .
$$

$L$ discharges through $R$ and $D_{3}$ remains ON. This gives us

$$
\frac{d i_{L}}{d t}=-\frac{1}{L} v_{C}, \frac{d v_{C}}{d t}=\frac{1}{C} i_{L}-\frac{1}{R C} v_{C} .
$$

The corresponding state space matrices are

$$
A_{2}=\left[\begin{array}{ccc}
0 & 0 & 0 \\
0 & 0 & -\frac{1}{L} \\
0 & \frac{1}{C} & -\frac{1}{R C}
\end{array}\right], B_{2}=\left[\begin{array}{c}
-\frac{n_{1}}{n_{2} L_{m}} \\
0 \\
0
\end{array}\right] .
$$




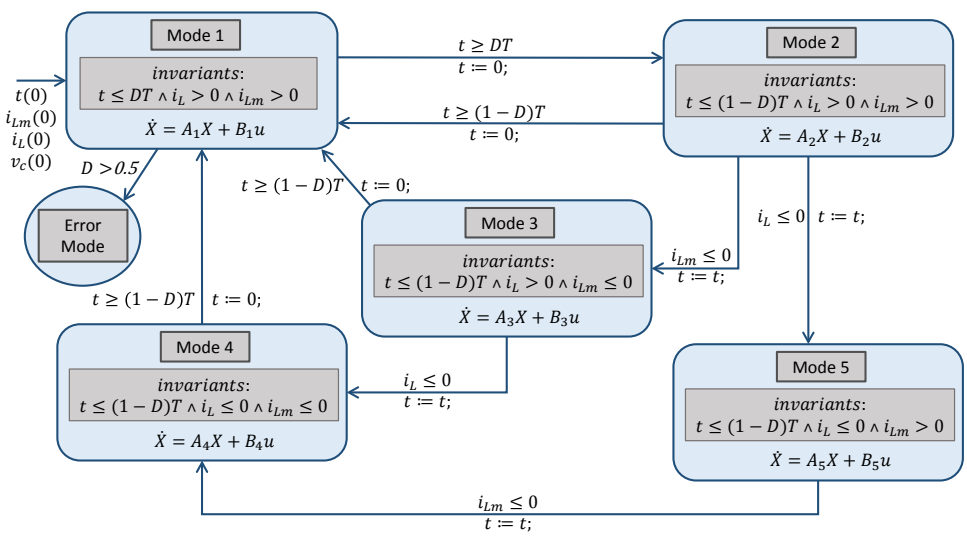

Figure 2.4: Hybrid automaton model for forward converter.

3. Mode 3: MOSFET switch is OFF such that $i_{L m} \leq 0$ and $i_{L}>0$, resulting

$$
\begin{gathered}
\frac{d i_{L m}}{d t}=0, \frac{d i_{L}}{d t}=-\frac{1}{L} v_{C}, \frac{d v_{C}}{d t}=\frac{1}{C} i_{L}-\frac{1}{R C} v_{C} . \\
A_{3}=\left[\begin{array}{ccc}
0 & 0 & 0 \\
0 & 0 & -\frac{1}{L} \\
0 & \frac{1}{C} & -\frac{1}{R C}
\end{array}\right], B_{3}=\left[\begin{array}{l}
0 \\
0 \\
0
\end{array}\right] .
\end{gathered}
$$

4. Mode 4: MOSFET switch is $\mathrm{OFF}, i_{L m} \leq 0$, and $i_{L} \leq 0$. This results

$$
\begin{gathered}
\frac{d i_{L m}}{d t}=0, \frac{d i_{L}}{d t}=0, \frac{d v_{C}}{d t}=-\frac{1}{R C} v_{C} . \\
A_{4}=\left[\begin{array}{ccc}
0 & 0 & 0 \\
0 & 0 & 0 \\
0 & 0 & -\frac{1}{R C}
\end{array}\right], B_{4}=\left[\begin{array}{l}
0 \\
0 \\
0
\end{array}\right] .
\end{gathered}
$$

5. Mode 5: MOSFET switch is OFF, $i_{L m}>0$, and $i_{L} \leq 0$. This gives us

$$
\begin{gathered}
\frac{d i_{L m}}{d t}=-\frac{n_{1} V_{i n}}{n_{2} L_{m}}, \frac{d i_{L}}{d t}=0, \frac{d v_{C}}{d t}=-\frac{1}{R C} v_{C} . \\
A_{5}=\left[\begin{array}{ccc}
0 & 0 & 0 \\
0 & 0 & 0 \\
0 & 0 & -\frac{1}{R C}
\end{array}\right], B_{5}=\left[\begin{array}{c}
-\frac{n_{1}}{n_{2} L_{m}} \\
0 \\
0
\end{array}\right] .
\end{gathered}
$$

6. Error Mode: Inherently, the maximum possible duty cycle for the forward converter is $D \leq 0.5$. Accordingly, we have added the error mode in the model to accommodate any deadlocks due to wrong selection of parameters. 

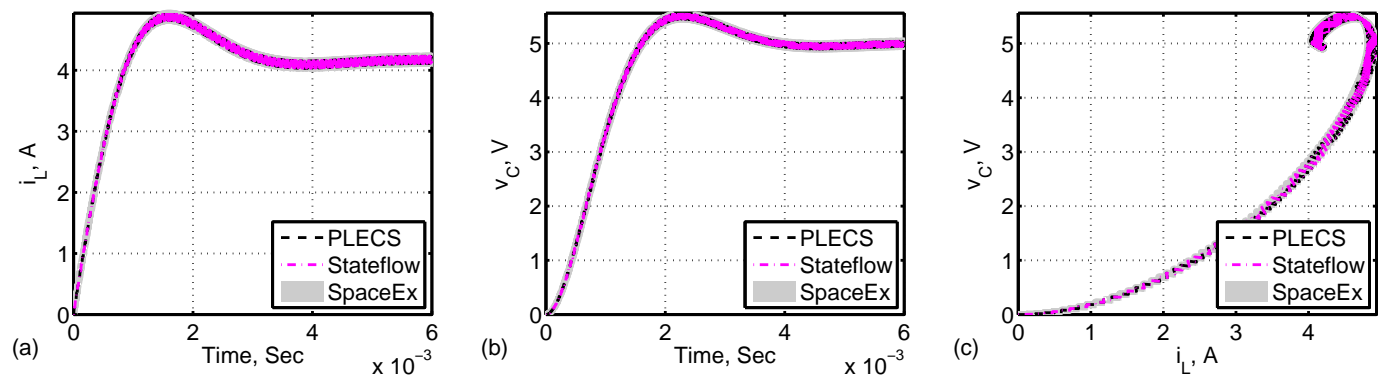

Figure 3.1: Comparison of SpaceEx reach sets, PLECS and SLSF trajectories for the flyback converter showing the simulation trace containment within overapproximated sets of reachable states: (a) Inductor current vs time (b) Capacitor voltage vs time (c) Phase-plane plot of capacitor voltage and inductor current.

Using the above ODEs and modes, the hybrid automaton model of forward converter is formulated and shown in Figure 2.4. The component values used in the model are mentioned in Figure 2.3 and adopted from [10].

\subsection{Closed-loop Forward Converter}

We have also modeled the forward converter in closed-loop configuration and typically used the hysteresis control methodology as outlined in [3]. In this control methodology, the capacitor voltage $v_{C}$ is allowed to vary within a hysteresis band. The hysteresis band is formed by defining an upper switching boundary, $V_{\text {ref }}+\Delta$, and a lower switching boundary, $V_{r e f}-\Delta$, where $V_{\text {ref }}$ is the desired output voltage, and $\Delta$ is the tolerance level. The state space description of the model remains the same as discussed in Section 2.3 and shown in Figure 2.2, whereas the guards $t \geq D T$ and $t \geq(1-D) T$ are changed to $v_{C} \geq V_{\text {ref }}+\Delta$ and $v_{C} \leq V_{\text {ref }}-\Delta$, respectively. Moreover, the invariants $t \leq D T$ and $t \leq(1-D) T$ are changed to $v_{C} \leq V_{\text {ref }}+\Delta$ and $v_{C} \geq V_{\text {ref }}-\Delta$, respectively.

\section{SLSF Simulations and Reachability Analysis}

We have automatically generated the hybrid automaton models in SpaceEx format using HyST tool and analyze these in SpaceEx environment. Moreover, we have automatically translated the same SpaceEx models into SLSF format using HyST. Formal verification of the flyback and forward converters includes verifying the corresponding capacitor voltage and inductor current to attain a stable limit cycle in settling time. For the flyback converter, we require that $v_{C}$ and $i_{L m}$ should exhibit a stable limit within settling time $t_{S}$. For the forward converter, we require that $v_{C}$ and $i_{L}$ should exhibit a stable limit within settling time $t_{S}$.

SpaceEx, PLECS, and SLSF results for the capacitor voltage and inductor current are shown in Figure 3.1. It is evident from the results in Figure 3.1 that PLECS and SLSF simulation traces are contained within the over-approximated sets of reachable states. We also conclude that these results exhibit stable limit cycle, and that stable voltage is attained within $5 \mathrm{~ms}$. 

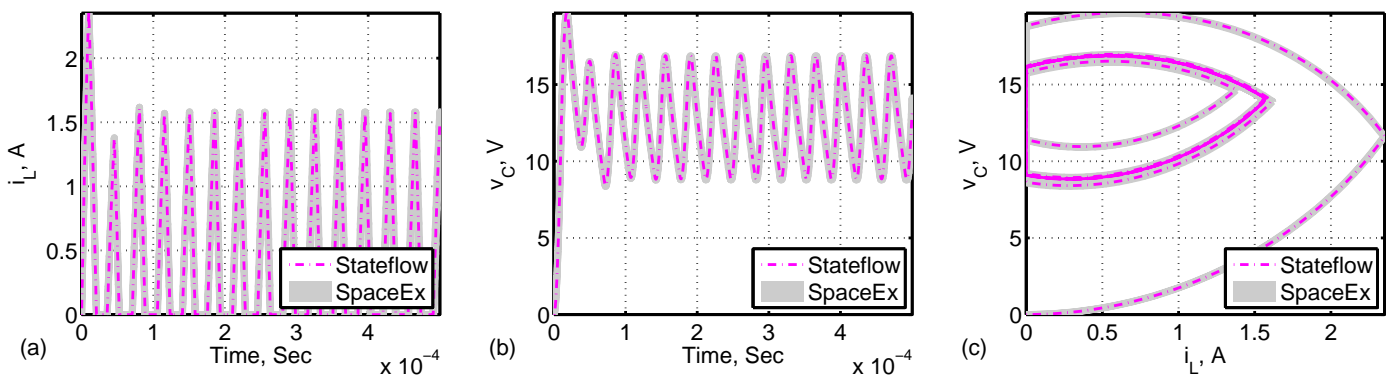

Figure 3.2: Comparison of SpaceEx overapproximations and SLSF trajectories for the open-loop forward converter, showing the simulation trace containment within overapproximated sets of reachable states: (a) Inductor current vs time (b) Capacitor voltage vs time (c) Phase-plane plot of capacitor voltage and inductor current.
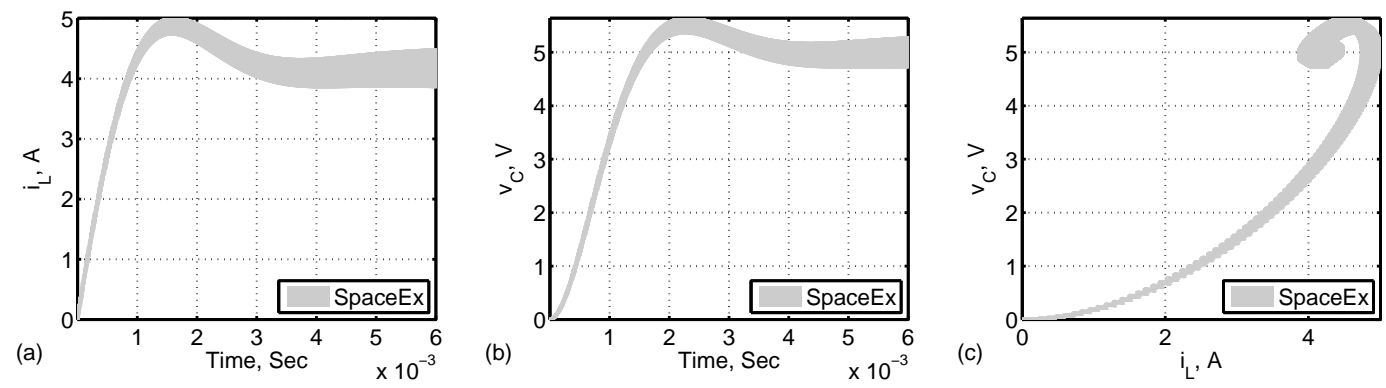

Figure 3.3: For the flyback converter model, we cater the non-determinism for the input voltage $V_{i n}$ and overapproximations are computed using SpaceEx: (a) Inductor current vs time (b) Capacitor voltage vs time (c) Phase-plane plot of capacitor voltage and inductor current.

We perform the reachability analysis using SpaceEx for forward converter as shown in Figure 3.2. The SLSF time traces are contained within the over-approximated sets of reachable states computed using SpaceEx. We also conclude that these results exhibit a stable limit cycle within $100 \mu \mathrm{s}$.

There are various sources of non-determinism in both the models such as the input voltage $\left(V_{i n}\right)$, initialization values of various state variables, the duty cycle of the PWM signal $(D)$, and the time period of PWM signals $(T)$. We have modeled the non-determinism of these parameters for both types of converters.

\subsection{Reachability Analysis Results - Non-Determinism in Flyback Converter}

First we consider the non-determinism in $V_{\text {in }}$ for the flyback converter, such that it is allowed to vary from $11.9-12.1 \mathrm{~V}$. The reachability analysis results are computed using SpaceEx and shown in Figure 3.3. We consider the variations in initial values of all the states variables, i.e., $i_{L m}$ and $v_{C}$. The state variable $i_{L m}$ is initialized for a range of $0-0.5 \mathrm{~A}$, whereas $v_{C}$ is initialized for $0-0.5 \mathrm{~V}$. The reachability analysis results are computed using SpaceEx and shown in Figure 3.4. Next we consider non-determinism in $D$, such that it is allowed to vary 

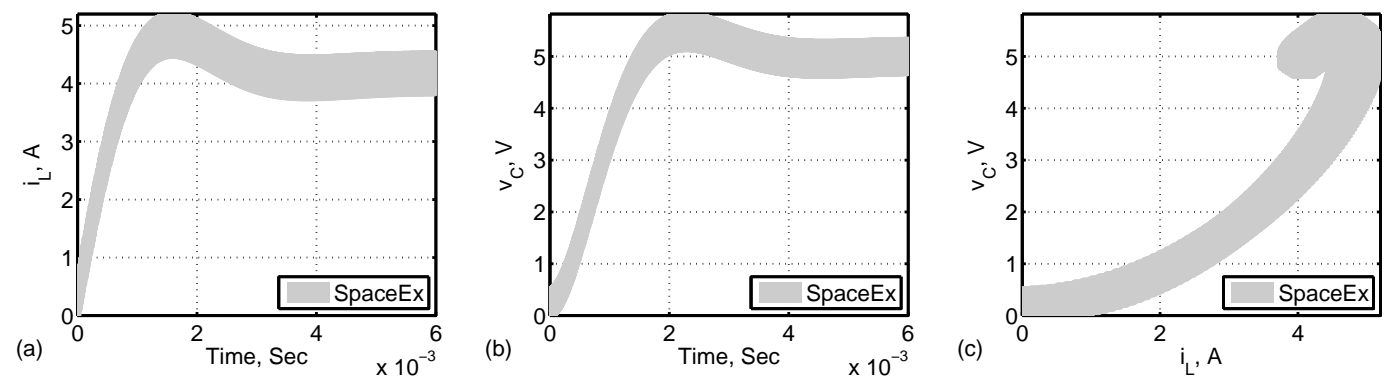

Figure 3.4: For the flyback converter model, we cater the non-determinism in initial values of $i_{L m}$ and $v_{C}$ and overapproximations are computed using SpaceEx: (a) Inductor current vs time (b) Capacitor voltage vs time (c) Phase-plane plot of capacitor voltage and inductor current.
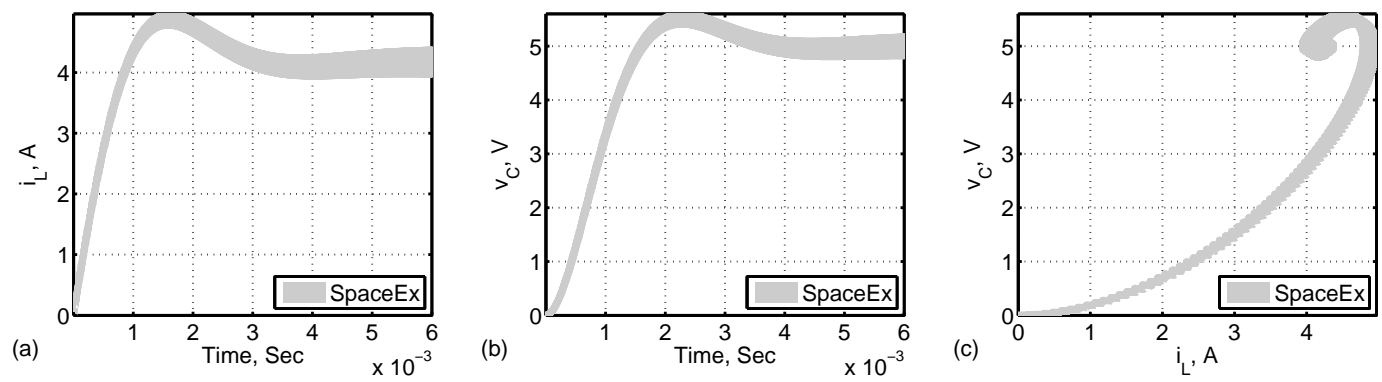

Figure 3.5: For the flyback converter model, we cater the non-determinism in the duty cycle $D$ and overapproximations are computed using SpaceEx: (a) Inductor current vs time (b) Capacitor voltage vs time (c) Phase-plane plot of capacitor voltage and inductor current.

from $0.449-0.501 \mathrm{~s}$. The overapproximations computed using SpaceEx are shown in Figure 3.5. In the last, we consider the variations in $T$ and obtain the reachability analysis results using SpaceEx as $T$ varies between $19.96-20.04 \mu s$, as shown in Figure 3.6.
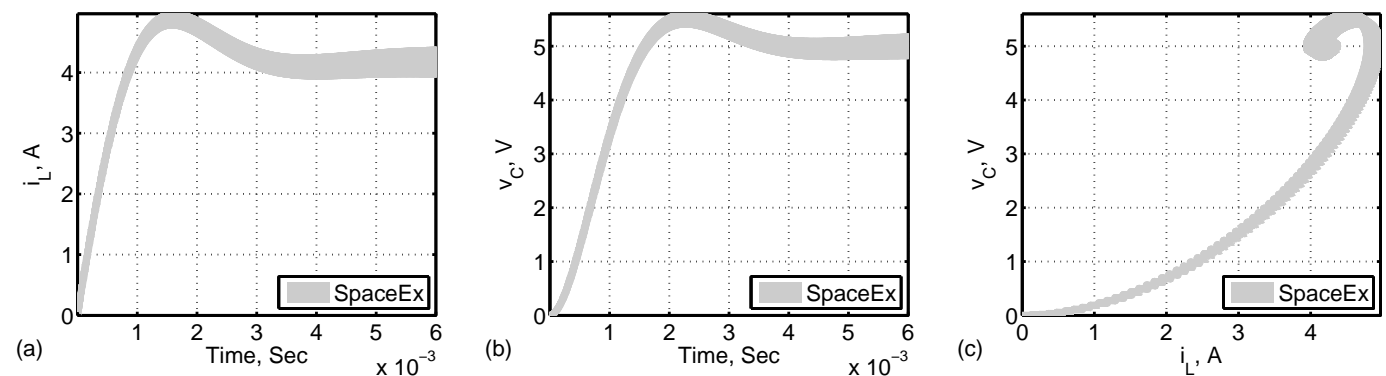

Figure 3.6: For the flyback converter model, we cater the non-determinism in the sampling time $T$ and overapproximations are computed using SpaceEx: (a) Inductor current vs time (b) Capacitor voltage vs time (c) Phase-plane plot of capacitor voltage and inductor current. 

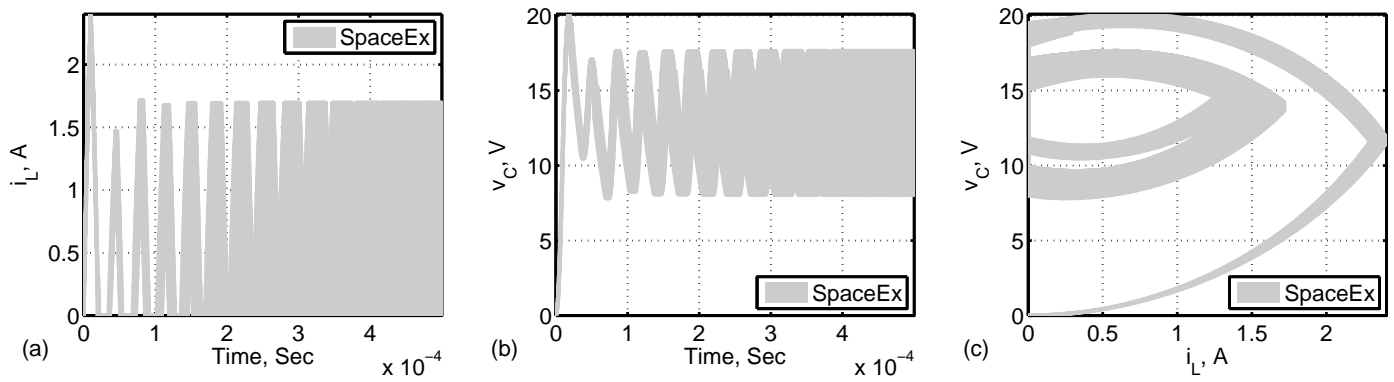

Figure 3.7: For the forward converter model, we cater the non-determinism for the input voltage $V_{i n}$ and overapproximations are computed using SpaceEx: (a) Inductor current vs time (b) Capacitor voltage vs time (c) Phase-plane plot of capacitor voltage and inductor current.
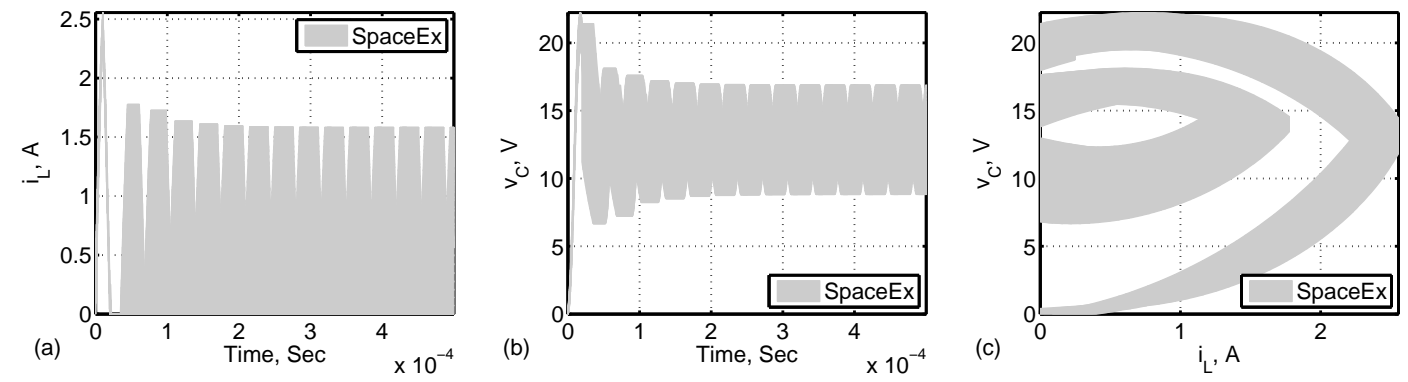

Figure 3.8: For the forward converter model, we cater the non-determinism in initial values of $i_{L}, i_{L m}$ and $v_{C}$ and overapproximations are computed using SpaceEx: (a) Inductor current vs time (b) Capacitor voltage vs time (c) Phase-plane plot of capacitor voltage and inductor current.

\subsection{Reachability Analysis Results - Non-Determinism in Forward Converter}

We consider the non-determinism in $V_{i n}$ for the forward converter, such that it is allowed to vary from $98-102 \mathrm{~V}$. The reachability analysis results are computed using SpaceEx and shown in Figure 3.7. We model the variations in initial values of all the states variables, i.e., $i_{L m}, i_{L}$, and $v_{C}$. The state variables $i_{L m}$ and $i_{L}$ are both initialized for a range of $0-0.4 \mathrm{~A}$, and $v_{C}$ is initialized for $0-0.4 \mathrm{~V}$. The reachability analysis results are computed using SpaceEx and shown in Figure 3.8. Next we consider non-determinism in $D$, such that it is allowed to vary from $0.39-0.41 s$. The overapproximations computed using SpaceEx are shown in Figure 3.9. In the last, we consider the variations in $T$ and obtain the reachability analysis results using SpaceEx as $T$ varies between $24.39-25.64 \mu s$, as shown in Figure 3.10.

\subsection{Reachability Analysis Results - Closed-loop Forward Converter}

In the last part, we present the reachability analysis results for the closed-loop forward converter using hysteresis control in Figure 3.11. For the hystersis-controlled forward converter we require that $i_{L}$ and $v_{C}$ should exhibit a stable limit cycle within the settling time $t_{S}$. As evident in Figure 3.11, both $i_{L}$ and $v_{C}$ exhibit a stable limit cycle within $50 \mu \mathrm{s}$. 

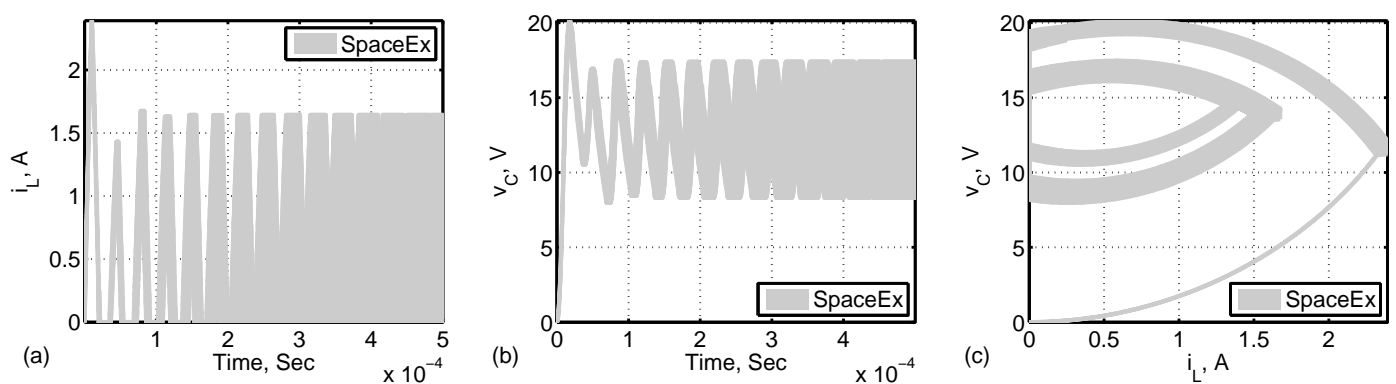

Figure 3.9: For the forward converter model, we cater the non-determinism in the duty cycle $D$ and overapproximations are computed using SpaceEx: (a) Inductor current vs time (b) Capacitor voltage vs time (c) Phase-plane plot of capacitor voltage and inductor current.
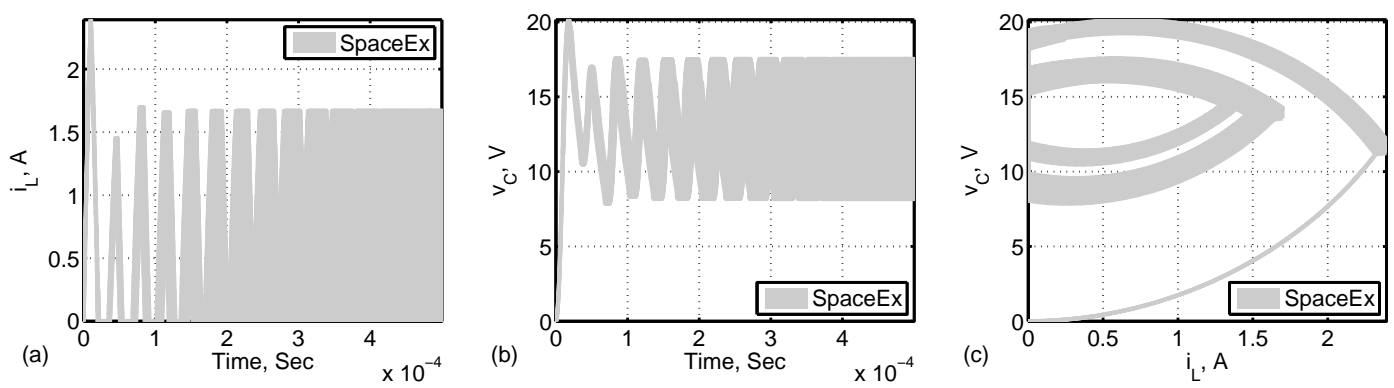

Figure 3.10: For the forward converter model, we cater the non-determinism in the sampling time $T$ and overapproximations are computed using SpaceEx: (a) Inductor current vs time (b) Capacitor voltage vs time (c) Phase-plane plot of capacitor voltage and inductor current.
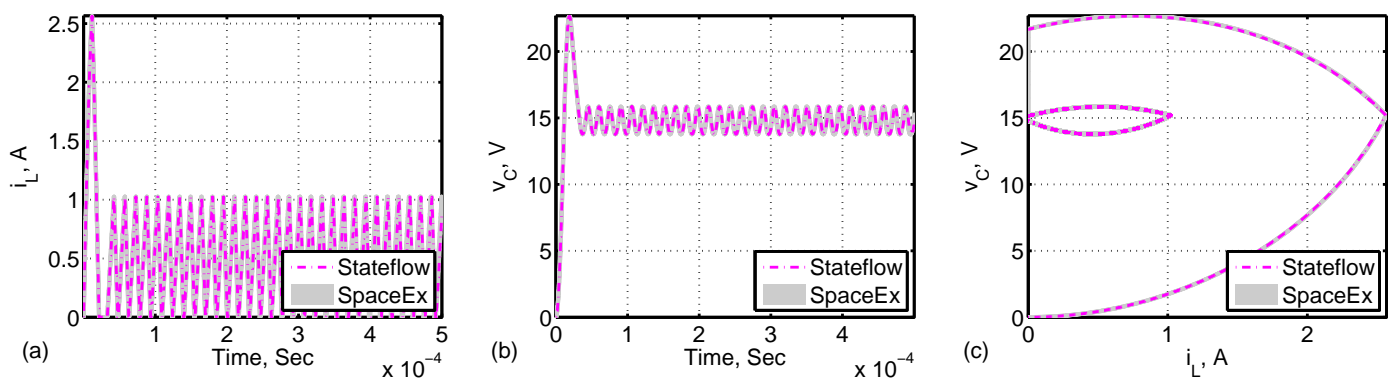

Figure 3.11: Comparison of SpaceEx and SLSF results for the hysteresis-controlled forward converter, showing the simulation trace containment within overapproximated sets of reachable states: (a) Inductor current vs time (b) Capacitor voltage vs time (c) Phase-plane plot of capacitor voltage and inductor current. 


\section{Key Observations}

Hybrid automaton modeling and reachability analysis of transformer-isolated flyback converter has medium difficulty level. However, modeling and analysis of forward converter is more complex with three state variables and five modes. We have only used SpaceEx to perform the reachability analysis. In addition other tools may also be used for the reachability analysis.

We have not considered the parasitics in modeling of transformer-isolated DC-DC converters that will further increase the difficulty level of this benchmark.

Moreover, we only consider a single DC-DC converter for analysis in the case studies. The reachability analysis for a group of such converters interacting with each other (e.g., in a DC microgrid) will pose a real challenge to the formal verification community.

\section{Benchmark Outlook}

On the whole, these verification benchmarks can serve as a first step towards a benchmark library to evaluate reachability and verification methods for various types of DC-DC converters. These benchmarks are open to the continuous and hybrid systems verification community to evaluate their methods and tools.

Acknowledgments The material presented in this paper is based upon the work supported by the National Science Foundation (NSF) under grant numbers ECCS 1137354, CNS 1464311, and CCF 1527398, the Air Force Research Laboratory (AFRL) through contract number FA8750-15-1-0105, and the Air Force Office of Scientific Research (AFOSR) under contract number FA9550-15-1-0258. The U.S. government is authorized to reproduce and distribute reprints for Governmental purposes notwithstanding any copyright notation thereon. Any opinions, findings, and conclusions or recommendations expressed in this publication are those of the authors and do not necessarily reflect the views of AFRL, AFOSR, or NSF.

The authors would also like to thank the anonymous reviewers whose valuable suggestions have added value to these benchmarks and enabled us to improve upon the results.

\section{References}

[1] Matthias Althoff, Akshay Rajhans, Bruce H. Krogh, Soner Yaldiz, Xin Li, and Larry Pileggi. Formal verification of phase-locked loops using reachability analysis and continuization. Commun. ACM, 56(10):97-104, October 2013.

[2] Stanley Bak, Sergiy Bogomolov, and Taylor T Johnson. Hyst: a source transformation and translation tool for hybrid automaton models. In Proceedings of the 18th International Conference on Hybrid Systems: Computation and Control, pages 128-133. ACM, 2015.

[3] Omar A. Beg, Houssam Abbas, Taylor T. Johnson, and Ali Davoudi. Model validation of pwm dc-dc converters. IEEE Transactions on Industrial Electronics, 2017. DOI: 10.1109/TIE.2017.2688961.

[4] Dae-Kyu Choi et al. A novel power conversion circuit for cost-effective battery-fuel cell hybrid systems. Journal of Power Sources, 152:245 - 255, 2005.

[5] Goran Frehse et al. Spaceex: Scalable verification of hybrid systems. In Shaz Qadeer Ganesh Gopalakrishnan, editor, Proc. 23rd International Conference on Computer Aided Verification (CAV), LNCS. Springer, 2011. 
[6] Shamina Hossain, Sairaj Dhople, and Taylor T. Johnson. Reachability analysis of closed-loop switching power converters. In Power and Energy Conference at Illinois (PECI), pages 130-134, 2013.

[7] Taylor T. Johnson, Zhihao Hong, and A. Kapoor. Design verification methods for switching power converters. In Power and Energy Conference at Illinois (PECI), 2012 IEEE, pages 1-6, February 2012.

[8] Luan Viet Nguyen and Taylor T. Johnson. Benchmark: Dc-to-dc switched-mode power converters (buck converters, boost converters, and buck-boost converters). In Applied Verification for Continuous and Hybrid Systems Workshop (ARCH 2014), Berlin, Germany, April 2014.

[9] Plexim Inc., Cambridge, MA, USA. PLECS Manual Version 4.0.4, 2016.

[10] A. Taut et al. Educational matlab tool for simulating of forward converters. In Proceedings of International Spring Seminar on Electronics Technology, pages 641-644, May 2011.

[11] Toyota. Defect information report (nhtsa recall 14v-053), Feb. 122014.

[12] Toyota. Defect information report (nhtsa recall 15v-449), Jul. 152015. 